\title{
Perfil antropométrico de crianças segundo a estrutura das creches
}

\author{
Anthropometric profile of children in accordance \\ with the structure of daycare centers
}

Dixis Figueroa Pedraza ${ }^{1}$

${ }^{1}$ Departamento de Enfermagem, Programa de Pós-Graduação em Saúde Pública, Universidade Estadual da Paraíba. Av. Baraúnas 351, Bodocongó. 58109-753 Campina Grande PB Brasil. dixisfigueroa@gmail.com
Abstract The scope of this article is to evaluate the structure of public daycare centers and the anthropometric profile of children in the city of Campina Grande in the State of Paraíba, Brazil. It is a cross-sectional study that involved 793 children attended in daycare centers. Information to describe the daycare centers were included (structure related to the capacity of demand and the processing power of school meals, reception system of children, location area) and the anthropometric status of the children (Height/Age, Weight/ Height). The mean Z-scores for Height/Age and Weight/Height were lower among children from daycare centers with a greater number of children per employee, attended on a part time basis and located in the rural zone. The Z-scores for Height/ Age were also lower among children who studied in classrooms and were housed in rest rooms with a greater density of children. Problems were detected in the structure of daycare centers with respect to the capacity of human resources to look after the children and the ways of living together (coexistence), which can make a difference in the nutritional status of the children. For its part, full-time daycare attendance and urbanization predispose children positively to growth.

Key words Child daycare centers, Children, Anthropometry, Body height, Growth
Resumo $O$ artigo tem por objetivos avaliar a estrutura de creches públicas e o perfil antropométrico de crianças do município de Campina Grande, Paraíba. Estudo transversal que envolveu 793 crianças assistidas em creches. Contemplaram-se informações para caracterizar as creches (estrutura relacionada à capacidade de atendimento à demanda e à de processamento da alimentação escolar, regime de acolhida, zona de localização) e o estado antropométrico das crianças (Estatura/Idade, Peso/Estatura). Menores escores- $Z$ de Estatura/Idade e Peso/Estatura apresentaram-se nas crianças que frequentavam creches com maior número de crianças por funcionário, em regimes de acolhida de tempo parcial e localizadas na zona rural. As crianças que conviviam em salas de aula e em salas de descanso com maior número de crianças apresentaram, também, menores escores- $Z$ de Estatura/Idade. Constatam-se problemas na estrutura das creches relacionados à capacidade de recursos humanos para prestar atenção às crianças e aos modos de convivência (aglomeração), os quais podem marcar diferenças no estado nutricional. Por sua vez, a frequência à creche em tempo integral e a urbanização predispõem positivamente o crescimento das crianças.

Palavras-chave Creches, Criança, Antropometria, Estatura, Crescimento 


\section{Introdução}

As creches públicas constituem um espaço privilegiado no qual parcela importante das crianças brasileiras em idade pré-escolar passam a maior parte do seu dia. Essas instituições abrigam, principalmente, crianças procedentes de famílias de vulnerabilidade socioeconômica dos centros urbanos $^{1,2}$. As creches têm um papel muito importante no desenvolvimento integral - físico, psicológico, intelectual e social - da criança ${ }^{2,3}$. Além dos cuidados oferecidos, a creche proporciona a oportunidade de promover estilos de vida saudáveis, inclusive na alimentação. Essas características e o acesso à alimentação fazem destas instituições um importante instrumento de garantia da segurança alimentar e nutricional, reafirmandose como proposta de política pública nos setores de educação, nutrição e saúde $e^{1,2,4,5}$.

Reconhecendo a importância das creches no cuidado das crianças, estudos desenvolvidos no Brasil têm enfocado o acesso, o estado e a evolução nutricional destas, o consumo de alimentos e o valor nutricional das refeições ${ }^{2}$. No entanto, são escassos os estudos com enfoque no papel dos profissionais e nas características/estrutura das instituições.

A importância dos profissionais que atuam nas creches deriva das necessidades que as crianças têm de cuidados adequados. A adoção de práticas de cuidado adequadas à manutenção e à recuperação da saúde infantil depende do perfil dos profissionais que atuam nas creches, o que evidencia a necessidade de formação direcionada à capacidade de prestar cuidados ${ }^{2}$.

A infraestrutura das creches, por sua vez, representa um importante indicador da sua qualidade $^{6}$. Assim, a legislação pertinente aplicada às creches também inclui abordagens sobre aspectos estruturais e recursos para a alimentação escolar ${ }^{7}$. Aspectos estruturais para o adequado funcionamento das creches foram atualizados na legislação brasileira por meio da Portaria n. ${ }^{0}$ 262/2011 do Ministério da Solidariedade e da Segurança Social, que preconiza normas reguladoras das condições de instalação e funcionamento ${ }^{8}$. Além disso, no contexto das novas diretrizes do Programa de Alimentação Escolar, fundamenta-se, por lei, a relevância de destinar recursos para a garantia do funcionamento e melhoria da infraestrutura física e pedagógica dos estabelecimentos de ensino?.

Em relação ao estado nutricional, as pesquisas apontam que crianças que frequentam creches e pertencem a estratos socioeconômicos menos favorecidos apresentam melhor condição nutricional do que aquelas da mesma condição que não fazem uso do benefício ${ }^{2}$. A literatura sugere, ainda, evolução nutricional positiva nas crianças que frequentam creches $^{10}$ e melhoras do estado nutricional daquelas com déficit nutricional quando aumenta o tempo de frequência na instituição ${ }^{2}$.

No entanto, não existem estudos não observacionais no Brasil que avaliem o impacto das creches. Nessa perspectiva, um trabalho de revisão da literatura identificou somente seis estudos, todos na América Latina, que avaliaram o impacto de programas de cuidado na saúde, na nutrição e no desenvolvimento de crianças menores de cinco anos de idade de países em desenvolvimento. Os autores advertiram para a impossibilidade de concluir sobre o impacto dos programas, reforçando a importância do desenvolvimento de novos estudos em cenários diferentes. Indicaram, ainda, que as pesquisas devem compreender a análise de variáveis intermediárias, como as características dos programas ou dos cuidados, que ajudem nas explicações das vias de impacto ${ }^{11}$. Estabelece-se, assim, a necessidade de estudos no Brasil que possam gerar hipóteses relacionadas ao estado nutricional das crianças, considerando as características das creches e seus serviços.

O presente estudo tem como objetivo avaliar a estrutura de creches públicas e o perfil antropométrico de crianças do município de Campina Grande, Paraíba.

\section{Metodologia}

Trata-se de um estudo transversal, integrado ao projeto "Segurança alimentar e nutricional de famílias com crianças menores de cinco anos do município de Campina Grande, Paraíba”. A coleta de dados aconteceu no período de outubro a novembro de 2011, em creches públicas do município de Campina Grande, Paraíba, pertencentes à Secretaria de Educação. Ao todo, funcionavam, no momento da coleta de dados, 25 creches em bairros distintos do município, situadas, geralmente, em áreas carentes. Segundo a localização, 23 creches estavam na zona urbana e duas na rural.

O universo de estudo foi de 2649 famílias cadastradas, das quais 2417 na zona urbana e 232 na rural. A população elegível incluiu todas as famílias, exceto aquelas com crianças gêmeas ou adotadas e com mães de idade inferior a 18 anos. Esses critérios de exclusão foram estabelecidos 
considerando a oportunidade de separar a contribuição desses fatores no crescimento das crianças. No caso da gemelaridade, como forma de distinguir entre os efeitos de tendências recebidas no nascimento e aquelas impostas pelo ambiente. No caso da adoção, devido a possíveis diferenças em traços comportamentais/psicológicos. No caso da idade materna inferior a 18 anos, considerando as possíveis consequências dessa condição em termos de complicações no estado de saúde e nutrição do filho. Além disso, foram excluídos os casos de crianças com problemas físicos que dificultassem a avaliação antropométrica.

O cálculo para estimar o tamanho da amostra baseou-se no procedimento para descrição da proporção ${ }^{12}$. Considerou-se uma prevalência estimada (p) de insegurança alimentar de 52,3\% (prevalência de insegurança alimentar para o Estado da Paraíba segundo os resultados da Pesquisa Nacional por Amostra de Domicílios de $2004^{13}$ ), um erro amostral (d) de 3\%, um nível de $95 \%$ de confiança $\left(Z \alpha^{2}=1,96^{2}\right)$ e poder da amostra de $80 \%$. Assim, estimou-se a necessidade de estudar 760 famílias, por meio da fórmula

$$
\mathrm{n}=\frac{\mathrm{N}{ }^{*} \mathrm{Z}_{\alpha}{ }^{2 *} \mathrm{p}^{*} \mathrm{q}}{\mathrm{d} 2{ }^{*}(\mathrm{~N}-1)+\mathrm{Z}_{\alpha}{ }^{2 *} \mathrm{p}^{*} \mathrm{q}}
$$

Considerando que cinco creches estavam em reforma e que uma foi compreendida no estudo piloto, totalizando 772 famílias não disponíveis e 1877 disponíveis, decidiu-se estudar 40 famílias em cada uma das 19 creches incluídas no estudo. Essas famílias foram escolhidas de forma aleatória no mesmo dia do trabalho de campo. Para as famílias com crianças irmãs frequentando creches, apenas uma delas foi considerada como unidade de análise.

Foram considerados como recusas os casos de mães que não deram o consentimento para participar da pesquisa. Foram definidos como perdas se a criança não compareceu à creche, ou não estava acompanhada da mãe, e quando não foi possível realizar sua avaliação antropométrica.

Para este estudo, foram contemplados dados sobre características das creches e antropometria das crianças. A caracterização das creches considerou parâmetros estruturais, o regime de acolhida das crianças (classificado como integral, quando a criança permanecia oito horas na creche, ou parcial, para quatro horas) e a zona de localização. A estrutura incluiu informações sobre a capacidade de atendimento à demanda e a capacidade de processamento da alimentação escolar. Complementarmente, foram obtidas in- formações sobre atividades que devem integrar as ações do Programa Nacional de Alimentação Escolar e em relação ao perfil das merendeiras.

A avaliação da estrutura das creches, segundo a capacidade de atendimento à demanda, contemplou dados relativos ao número de crianças por funcionário (total de crianças na creche/total de funcionários na creche); à média de crianças convivendo na mesma sala de aula, por creche; e à média de crianças convivendo na mesma sala de descanso, por creche. Essas variáveis foram qualificadas, considerando como ponto de corte a média do parâmetro respectivo para o total de creches, em duas categorias $(<=$ média do total de creches ou $>$ média do total de creches).

A capacidade de processamento da alimentação escolar analisou-se segundo oito critérios $^{14}$ : existência de merendeira, área da cozinha, condições em relação aos equipamentos principais (fogão, geladeira, freezer), condições em relação aos equipamentos auxiliares (liquidificador, batedeira), disponibilidade de utensílios de preparo, disponibilidade de utensílios para servir, existência de refeitório, existência de despensa. Todos os critérios admitiram valores de 0,1 ou 2 . Os critérios merendeira, equipamentos principais e equipamentos auxiliares admitiram, também, valores de 0,5 ou 1,5, dependendo da composição da equipe de merendeiras e do estado de funcionamento dos equipamentos. A capacidade de processamento da merenda escolar foi qualificada segundo o escore total: $<8$ (insatisfatório), 8-12 (regular), 13-16 (satisfatório).

Como parte da descrição relativa às atividades que devem integrar o Programa Nacional de Alimentação Escolar, obtiveram-se informações sobre a existência de horta escolar, o desenvolvimento de ações de saúde e nutrição, a participação dos pais e a supervisão do Programa. Para esses fins, os professores e diretores das creches responderam um questionário e as variáveis foram sumarizadas por frequência simples.

O perfil das merendeiras com tempo de serviço de seis meses ou mais englobou dados sobre características do trabalho (tempo na profissão, dedicação parcial ou integral, função principal, tipo de vínculo, desenvolvimento de outras atividades, recebimento de horas extras, experiência prévia) e afinidade com a profissão (motivo para trabalhar como merendeira - por necessidade ou por opção -, prazer em ser merendeira, preparação para exercer a profissão e interesse em aperfeiçoamento). As variáveis originadas do questionário aplicado às merendeiras, para esses fins, foram sumarizadas por frequência simples. 
As medidas de estatura e peso das crianças foram obtidas obedecendo às normas técnicas padronizadas recomendadas pela Organização Mundial da Saúde ${ }^{15}$. As medições foram realizadas, sempre na presença da mãe, em duplicata, aceitando-se variação de $0,3 \mathrm{~cm}$ para medida de estatura e $100 \mathrm{~g}$ para medida de peso. Se esses limites fossem eventualmente ultrapassados, repetia-se a mensuração, anotando-se as duas medições com valores mais próximos, utilizando sua média para efeito de registro.

As crianças menores de dois anos tiveram o comprimento medido (criança deitada) por meio de antropômetro infantil de madeira (Alturexata ${ }^{\circledast}$ com amplitude de $130 \mathrm{~cm}$ e subdivisões de $0,1 \mathrm{~cm}$. As crianças de dois anos ou mais tiveram a altura medida (criança em pé) por meio de estadiômetro (WCS ${ }^{\varpi}$ ) com amplitude de $200 \mathrm{~cm}$ e subdivisões de $0,1 \mathrm{~cm}$. Na medição, a criança foi posicionada no centro do instrumento, descalça e com a cabeça livre de adereços; a qual foi apoiada firmemente contra a parte fixa do equipamento, com o pescoço reto e o queixo afastado do peito, no plano de Frankfurt; braços estendidos ao longo do corpo; ombros, nádegas e calcanhares em contato com a superfície; joelhos levemente pressionados para baixo de modo a ficarem estendidos; pés juntos, fazendo um ângulo reto com as pernas.

Todas as crianças foram pesadas com a utilização de balança eletrônica do tipo plataforma com capacidade para $150 \mathrm{~kg}$ e graduação em 100g (Tanita UM- $080^{\varpi}$ ). Na pesagem, a criança foi posicionada no centro do equipamento, permitindo-se apenas uma peça íntima leve, as quais, no caso das que usavam fraldas, foram retiradas. O peso das crianças de colo foi calculado pela diferença entre o peso da mãe com a criança no colo e o peso da mãe.

Os escores-Z de Estatura/Idade e Peso/Estatura das crianças foram calculados com o programa WHO Anthro 2009. Tomou-se como referência a população do Multicentre Growth Reference $S t u d y^{16}$, recomendação atual da Organização Mundial da Saúde e do Ministério da Saúde do Brasil.

Com o objetivo de assegurar a validação da digitação, os dados foram digitados com dupla entrada, através do programa Excel (Microsoft Inc., Estados Unidos). Após o término da digitação, os dois bancos de dados foram cruzados com a utilização do aplicativo Validate do programa Epi Info v. 6.04b (WHO/CDC, Atlanta, Estados Unidos). Possibilitou-se, assim, verificar a consistência dos dados e gerar o banco final que foi usado nas análises estatísticas. Para testar a suposição de normalidade das variáveis envolvidas no estudo, foi aplicado o teste de Shapiro Wilk.

Neste trabalho, os berçários de duas creches foram considerados de forma independente, totalizando 21 unidades de estudo, as quais foram identificadas pelas letras da $\mathrm{A}$ à $\mathrm{U}$.

O teste $\mathrm{t}$ de Student foi utilizado para verificar diferenças entre as médias de escores- $Z$ de Estatura/Idade e Peso/Estatura segundo as características das creches. As análises estatísticas foram realizadas através do programa Rv2.10.0., considerando-se significância estatística um valor $\mathrm{p}<0,05$.

O projeto foi aprovado pelo Comitê de Ética em Pesquisa da Universidade Estadual da Paraíba. Todas as mães cujas crianças foram avaliadas e as diretoras das creches assinaram o Termo de Consentimento Livre e Esclarecido. Os resultados foram divulgados nas instâncias pertinentes através de encontros com a Secretaria de Educação do município e por meio de contatos pessoais com os pais ou responsáveis pelas crianças. A divulgação compreendeu o diagnóstico do município, por creche e por criança.

\section{Resultados}

Das 1877 famílias disponíveis para o estudo, 1728 foram consideradas elegíveis. Foram excluídas 74 famílias com crianças gêmeas, 47 com crianças adotadas, 14 com crianças com problemas físicos que comprometeriam a avaliação antropométrica e 14 com mães menores de 18 anos. Em 23 casos, as mães recusaram participar da pesquisa. Totalizaram-se 83 perdas, 64 por crianças que não compareceram à creche ou não estavam acompanhadas de suas mães no dia da coleta de dados e 19 por crianças nas quais foi impossível realizar a avaliação antropométrica.

Devido ao porte de algumas creches, foi impossível atingir a amostra proposta de 40 famílias por unidade. Contudo, esta incompletude foi recompensada com o estudo de maior quantidade de famílias nas creches de grande porte, totalizando as 760 do tamanho amostral. Além disso, ao final da coleta dos dados, uma das creches, que estava em reforma, tinha recomeçado suas atividades, sendo incluída na coleta de dados com a obtenção de informações de 33 famílias. Assim, foram analisados os dados correspondentes a 793 famílias com crianças assistidas em creches.

Os itens analisados na capacidade de atendimento à demanda (Tabela 1) mostraram varia- 
Tabela 1. Indicadores da capacidade de atendimento à demanda em creches públicas municipais. Campina Grande, Paraíba, 2011.

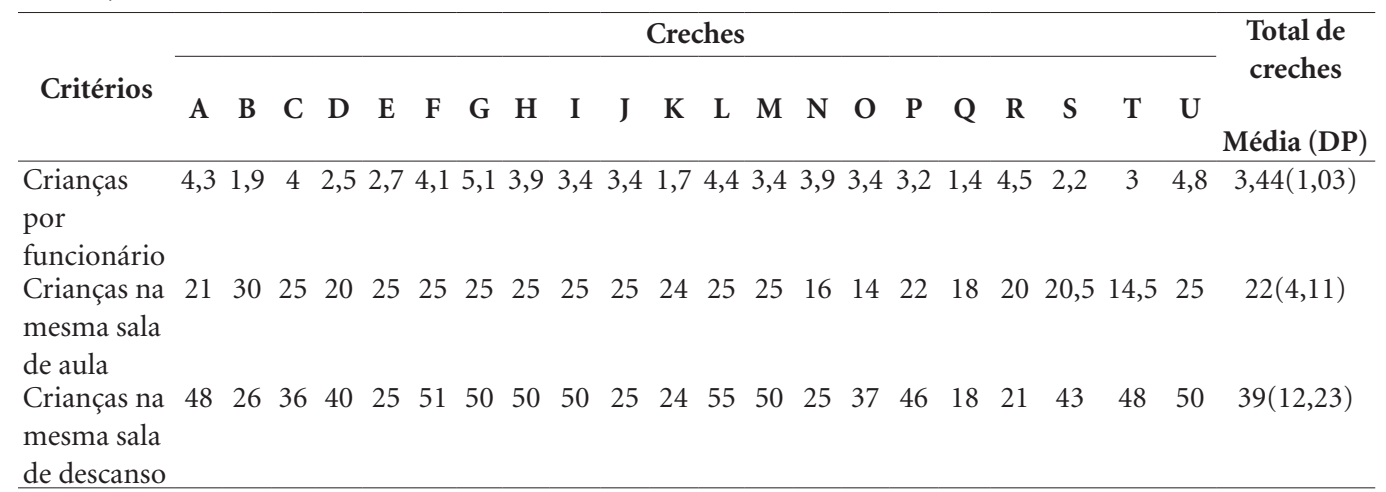

Crianças por funcionário: total de crianças na creche/total de funcionários na creche; crianças na mesma sala de aula: média de crianças que conviviam na creche na mesma sala de aula; crianças na mesma sala de descanso: média de crianças que conviviam na creche na mesma sala de descanso.

ções expressivas, de 1,4 a 4,4 para o número de crianças por funcionário, de 14 a 30 para o número de crianças na mesma sala de aula e de 18 a 51 para o número de crianças na mesma sala de descanso. Podem-se observar desvantagens estruturais em algumas creches, a exemplo da "Q".

Os resultados da capacidade de processamento da alimentação escolar constam na Tabela 2. Conforme se pode verificar, a avaliação média foi satisfatória, estando somente duas creches classificadas na categoria regular. Os itens equipamentos auxiliares, merendeira e equipamentos principais foram os mais comprometidos, enquanto a despensa foi o item de melhor avaliação.

Em relação às atividades que devem integrar o Programa de Alimentação Escolar, evidenciouse, em geral, deficiência. Constataram-se apenas duas creches com horta escolar. Alguma atividade de saúde (participação eventual do dentista da Estratégia Saúde da Família com atividades educativas e de saúde bucal) foi reportada pelos diretores de seis creches. Ações de saúde e nutrição desenvolvidas pelos professores na sala de aula foram referidas por sete deles. A participação dos pais foi indicada por quatro diretores, os quais informaram o desenvolvimento de reuniões para a apresentação dos cardápios e abordagens sobre o tema alimentação. Em todas as creches, os diretores afirmaram existir supervisão do Programa, realizada por uma empresa terceirizada com a participação de nutricionista.

Foram realizadas 61 entrevistas com merendeiras de idade entre 24-61 anos (média de 41,5 anos) e tempo de trabalho nessa profissão entre seis meses e 30 anos (média de 5,8 anos). Do to- tal de merendeiras, 35 desenvolviam a função em tempo parcial e 26 em integral; 38 definiram-se como merendeiras e 23 como merendeiras com função de servente ou vice-versa; 39 tinham vínculo efetivo da prefeitura, 11 contrato da prefeitura e 11 contrato de empresa terceirizada (a mesma que realizava a supervisão do Programa). Houve merendeiras que declararam exercer outras atividades $(n=9)$, receber horas extras $(n=$ 14) e não ter trabalhado antes $(n=15)$.

Em relação à afinidade com a profissão, destaca-se que 30 profissionais afirmaram trabalhar como merendeira por opção e 31, por necessidade. Além disso, apenas três declararam não gostar da profissão. As principais referências que as merendeiras deram, como explicação do porque gostar do trabalho, foram o prazer em cozinhar/ preparar alimentos e em lidar com crianças. A respeito da formação na área de alimentação e nutrição, 14 merendeiras referiram nunca ter frequentado cursos (nutrição e higiene dos alimentos, manipulação de alimentos) da área. Por outro lado, 21 merendeiras manifestaram desinteresse em frequentar cursos ou treinamentos similares. Os cursos que gostariam de receber, mais frequentemente referidos pelas merendeiras, foram, na ordem, culinária e preparação de alimentos; higiene e manipulação de alimentos; e congelamento, conservação e aproveitamento dos alimentos. O tema sobre o valor nutricional dos alimentos e alimentação saudável também foi referido por algumas merendeiras.

As crianças estudadas apresentaram médias de escore-Z dos índices Estatura/Idade e Peso/ Estatura de $-0,42 \pm 1,09$ e $0,54 \pm 1,04$, respecti- 
Tabela 2. Indicadores da capacidade de processamento da alimentação escolar em creches públicas municipais. Campina Grande, Paraíba, 2011.

\begin{tabular}{|c|c|c|c|c|c|c|c|c|c|c|c|c|c|}
\hline \multirow{2}{*}{ Critérios } & \multicolumn{13}{|c|}{ Creches } \\
\hline & $\mathbf{A}$ & B & $\mathrm{C}$ & D & E & $\mathbf{F}$ & G & $\mathbf{H}$ & I & $\mathbf{J}$ & K & $\mathbf{L}$ & $\mathbf{M}$ \\
\hline Merendeira & 1,5 & 1 & 1 & 1 & 1,5 & 1 & 1 & 1,5 & 1,5 & 1,5 & 2 & 2 & 2 \\
\hline Área da cozinha & 2 & 2 & 2 & 2 & 2 & 2 & 2 & 1 & 1 & 2 & 2 & 2 & 2 \\
\hline Equipamentos principais & 1,5 & 2 & 2 & 0,5 & 0,5 & 1,5 & 2 & 1,5 & 1,5 & 1,5 & 1,5 & 1,5 & 2 \\
\hline Equipamentos auxiliares & 1 & 1 & 1 & 2 & 2 & 1 & 1 & 1 & 2 & 2 & 1,5 & 1,5 & 1,5 \\
\hline Utensílios de preparo & 1 & 2 & 2 & 2 & 2 & 2 & 2 & 2 & 2 & 2 & 2 & 2 & 2 \\
\hline Utensílios para servir & 1 & 2 & 2 & 1 & 2 & 2 & 2 & 2 & 2 & 2 & 2 & 2 & 1 \\
\hline Refeitório & 2 & 1 & 1 & 2 & 1 & 2 & 2 & 2 & 2 & 2 & 1 & 1 & 2 \\
\hline Despensa & 2 & 2 & 2 & 2 & 2 & 2 & 2 & 2 & 2 & 2 & 1 & 2 & 2 \\
\hline Escore & 12 & 13 & 13 & 12,5 & 13 & 13,5 & 14 & 13 & 14 & 15 & 13 & 14 & 14,5 \\
\hline Avaliação & $\operatorname{Re}$ & $\mathrm{Sa}$ & $\mathrm{Sa}$ & $\mathrm{Sa}$ & $\mathrm{Sa}$ & $\mathrm{Sa}$ & $\mathrm{Sa}$ & $\mathrm{Sa}$ & $\mathrm{Sa}$ & $\mathrm{Sa}$ & $\mathrm{Sa}$ & $\mathrm{Sa}$ & $\mathrm{Sa}$ \\
\hline \multirow[t]{2}{*}{ Critérios } & \multicolumn{9}{|c|}{ Creches } & \multicolumn{4}{|c|}{ Total de creches } \\
\hline & $\mathrm{N}$ & $\mathbf{O}$ & $\mathbf{P}$ & Q & $\mathbf{R}$ & $S$ & $\mathbf{T}$ & $\mathbf{U}$ & & \multicolumn{4}{|c|}{ Média (DP) } \\
\hline Merendeira & 1 & 1,5 & 2 & 2 & 2 & 1,5 & 2 & 1,5 & & \multicolumn{4}{|c|}{$1,52(0,40)$} \\
\hline Área da cozinha & 2 & 1 & 2 & 2 & 2 & 2 & 2 & 2 & & \multicolumn{4}{|c|}{$1,86(0,36)$} \\
\hline Equipamentos principais & 2 & 1,5 & 1,5 & 1,5 & 1,5 & 2 & 2 & 2 & & \multicolumn{4}{|c|}{$1,59(0,44)$} \\
\hline Equipamentos auxiliares & 2 & 1,5 & 0,5 & 1 & 2 & 2 & 2 & 2 & & \multicolumn{4}{|c|}{$1,50(0,50)$} \\
\hline Utensílios de preparo & 2 & 2 & 1 & 2 & 2 & 2 & 2 & 2 & & \multicolumn{4}{|c|}{$1,90(0,30)$} \\
\hline Utensílios para servir & 2 & 2 & 1 & 2 & 2 & 2 & 2 & 2 & & \multicolumn{4}{|c|}{$1,81(0,40)$} \\
\hline Refeitório & 2 & 2 & 1 & 2 & 2 & 2 & 2 & 2 & & \multicolumn{4}{|c|}{$1,71(0,46)$} \\
\hline Despensa & 2 & 2 & 2 & 2 & 2 & 2 & 2 & 2 & & \multicolumn{4}{|c|}{$1,95(0,22)$} \\
\hline Escore & 15 & 13,5 & 11 & 14,5 & 15,5 & 15,5 & 16 & 15,5 & & \multicolumn{4}{|c|}{$13,86(1,29)$} \\
\hline Avaliação & $\mathrm{Sa}$ & $\mathrm{Sa}$ & $\mathrm{Re}$ & $\mathrm{Sa}$ & $\mathrm{Sa}$ & $\mathrm{Sa}$ & $\mathrm{Sa}$ & $\mathrm{Sa}$ & & \multicolumn{4}{|c|}{$\mathrm{Sa}$} \\
\hline
\end{tabular}

Re: Regular / Sa: Satisfatório; os critérios de avaliação admitiram valores de 0, 1 ou 2; os critérios merendeira, equipamentos principais e equipamentos auxiliares admitiram, também, valores de 0,5 ou 1,5, dependendo da composição da equipe de merendeiras e do estado de funcionamento dos equipamentos; Escore: $<8$ (insatisfatório), 8-12 (regular), 13-16 (satisfatório).

vamente. As médias dos dois índices antropométricos apresentaram ampla variação entre as creches. A diferença entre a pior $(-1,15)$ e a melhor $(-0,11)$ média de escore-Z do índice Estatura/ Idade atingiu 1,04. Portanto, as crianças da creche $\mathrm{K}$ apresentaram 1,04 desvios padrão mais abaixo da mediana da população de referência do que as crianças da creche E (Tabela 3).

Os valores médios para os escores- $Z$ de Estatura/Idade e Peso/Estatura, de acordo com a Tabela 4, apresentaram-se inferiores nas crianças que frequentavam creches com maior número de crianças por funcionário, em regimes de acolhida de tempo parcial e localizadas na zona rural. As crianças que conviviam em salas de aula e em salas de descanso com maior número de crianças, apresentaram, também, menores escores- $Z$ de Estatura/Idade.

\section{Discussão}

A despeito das diversas contribuições teóricas e legais em relação à importância da estrutura das creches, poucos estudos da área de saúde têm buscado avaliar tais parâmetros e seus efeitos $^{6-9,11}$. Entretanto, na área de educação, observa-se maior importância atribuída ao tema ${ }^{17,18}$. Nesse sentido, cabe destacar dois estudos. O primeiro, uma revisão da literatura que constatou a precariedade das creches em relação à formação de pessoal e à infraestrutura material, com pior situação quando comparadas às pré-escolas ${ }^{17}$. 
Tabela 3. Avaliação antropométrica de crianças em creches públicas municipais. Campina Grande, Paraíba, 2011.

\begin{tabular}{|c|c|c|c|c|c|c|c|c|c|c|c|c|c|}
\hline \multirow[b]{2}{*}{ Índices } & \multicolumn{13}{|c|}{ Creches } \\
\hline & A & B & $\mathrm{C}$ & D & E & F & G & $\mathbf{H}$ & I & $\mathbf{J}$ & K & $\mathrm{L}$ & M \\
\hline \multicolumn{14}{|l|}{ E/I (escore-Z) } \\
\hline Média (DP) & $\begin{array}{l}-0,4 \\
(1,2)\end{array}$ & $\begin{array}{l}-0,5 \\
(1,9)\end{array}$ & $\begin{array}{l}-0,7 \\
(1,3)\end{array}$ & $\begin{array}{l}-0,2 \\
(0,9)\end{array}$ & $\begin{array}{l}-0,1 \\
(1,1)\end{array}$ & $\begin{array}{l}-0,7 \\
(1,0)\end{array}$ & $\begin{array}{l}-0,8 \\
(0,9)\end{array}$ & $\begin{array}{l}-0,2 \\
(1,1)\end{array}$ & $\begin{array}{l}-0,3 \\
(1,2)\end{array}$ & $\begin{array}{l}-0,5 \\
(0,8)\end{array}$ & $\begin{array}{l}-1,1 \\
(0,9)\end{array}$ & $\begin{array}{l}-0,6 \\
(1,1)\end{array}$ & $\begin{array}{l}-0,6 \\
(0,9)\end{array}$ \\
\hline \multicolumn{14}{|l|}{$\mathrm{P} / \mathrm{E}$ (escore-Z) } \\
\hline Média (DP) & $\begin{array}{c}0,6 \\
(1,0) \\
\end{array}$ & $\begin{array}{c}1,0 \\
(0,9)\end{array}$ & $\begin{array}{c}0,3 \\
(0,8)\end{array}$ & $\begin{array}{c}0,5 \\
(0,9) \\
\end{array}$ & $\begin{array}{c}0,8 \\
(1,3)\end{array}$ & $\begin{array}{c}0,5 \\
(0,8)\end{array}$ & $\begin{array}{c}0,3 \\
(0,9) \\
\end{array}$ & $\begin{array}{c}0,4 \\
(1,2)\end{array}$ & $\begin{array}{c}0,7 \\
(1,1) \\
\end{array}$ & $\begin{array}{c}0,3 \\
(0,9) \\
\end{array}$ & $\begin{array}{c}1,0 \\
(1,0) \\
\end{array}$ & $\begin{array}{c}0,4 \\
(1,0) \\
\end{array}$ & $\begin{array}{c}0,3 \\
(1,0)\end{array}$ \\
\hline & \multicolumn{10}{|c|}{ Creches } & \multirow{2}{*}{\multicolumn{3}{|c|}{$\begin{array}{l}\text { Total de creches } \\
\text { Média (DP) }\end{array}$}} \\
\hline Índices & $\mathrm{N}$ & O & & $\mathbf{P}$ & Q & $\mathbf{R}$ & $S$ & & $\mathrm{~T}$ & $\mathrm{U}$ & & & \\
\hline \multicolumn{14}{|l|}{ E/I (escore-Z) } \\
\hline Média (DP) & $\begin{array}{l}-0,2 \\
(1,1)\end{array}$ & $\begin{array}{l}-0,1 \\
(1,1)\end{array}$ & & $\begin{array}{l}0,5 \\
1,0)\end{array}$ & $\begin{array}{l}-0,2 \\
(1,2)\end{array}$ & $\begin{array}{l}-0,2 \\
(1,3)\end{array}$ & $\begin{array}{l}-0,3 \\
(1,0)\end{array}$ & & $\begin{array}{l}-0,3 \\
(0,8)\end{array}$ & $\begin{array}{l}-0,7 \\
(1,0)\end{array}$ & & $\begin{array}{l}-0,42 \\
(1,09)\end{array}$ & \\
\hline \multicolumn{14}{|l|}{$\mathrm{P} / \mathrm{E}$ (escore-Z) } \\
\hline Média (DP) & $\begin{array}{c}0,8 \\
(1,1)\end{array}$ & $\begin{array}{c}2,2 \\
(0,9)\end{array}$ & & $\begin{array}{l}0,5 \\
1,1)\end{array}$ & $\begin{array}{c}0,9 \\
(1,3)\end{array}$ & $\begin{array}{c}0,6 \\
(1,0)\end{array}$ & $\begin{array}{r}0,9 \\
(1,0)\end{array}$ & & $\begin{array}{c}0,7 \\
(1,2)\end{array}$ & $\begin{array}{c}1,0 \\
(0,9)\end{array}$ & & $\begin{array}{c}0,54 \\
(1,04)\end{array}$ & \\
\hline
\end{tabular}

E/I: Estatura/Idade; P/E: Peso/Estatura.

Tabela 4. Médias (DP) dos índices Estatura/Idade (escore-Z) e Peso/Estatura (escore-Z) de crianças assistidas em creches municipais segundo características das creches. Campina Grande, Paraíba, 2011.

\begin{tabular}{|c|c|c|c|c|c|c|}
\hline Características das creches & $\mathbf{n}$ & $\%$ & $\begin{array}{l}\text { Média (DP) de } \\
\text { Estatura/Idade } \\
\quad(\text { escore-Z) }\end{array}$ & p & $\begin{array}{l}\text { Média (DP) de } \\
\text { Peso/Estatura } \\
\text { (escore-Z) }\end{array}$ & $\mathbf{p}$ \\
\hline Crianças por funcionário & & & & 0,044 & & 0,030 \\
\hline$<=$ Média do total de creches & 321 & 40,5 & $-0,35(1,07)$ & & $0,64(1,10)$ & \\
\hline$>$ Média do total de creches & 472 & 59,5 & $-0,49(1,10)$ & & $0,48(1,00)$ & \\
\hline Crianças na mesma sala de aula & & & & 0,010 & & 0,500 \\
\hline$<=$ Média do total de creches $>$ & 353 & 44,5 & $-0,31(1,06)$ & & $0,57(1,06)$ & \\
\hline Média do total de creches & 440 & 55,5 & $-0,51(1,11)$ & & $0,52(1,03)$ & \\
\hline $\begin{array}{l}\text { Crianças na mesma sala de } \\
\text { descanso }\end{array}$ & & & & 0,023 & & 0,776 \\
\hline$<=$ Média do total de creches $>$ & 277 & 34,9 & $-0,30(1,18)$ & & $0,56(1,09)$ & \\
\hline Média do total de creches & 516 & 65,1 & $-0,49(1,03)$ & & $0,54(1,03)$ & \\
\hline ECPAE & & & & 0,602 & & 0,773 \\
\hline Satisfatório & 624 & 78,7 & $-0,38(1,10)$ & & $0,55(1,06)$ & \\
\hline Não satisfatório & 169 & 21,3 & $-0,43(1,05)$ & & $0,53(1,01)$ & \\
\hline Regime de acolhida na creche & & & & 0,000 & & 0,000 \\
\hline Tempo total & 408 & 51,5 & $-0,24(1,11)$ & & $0,72(1,00)$ & \\
\hline Tempo parcial & 385 & 48,5 & $-0,59(1,05)$ & & $0,36(1,06)$ & \\
\hline Zona de localização & & & & 0,022 & & 0,044 \\
\hline Urbana & 770 & 97,1 & $-0,12(1,05)$ & & $0,98(0,99)$ & \\
\hline Rural & 23 & 2,9 & $-0,30(1,01)$ & & $0,53(1,05)$ & \\
\hline
\end{tabular}

Crianças por funcionário: total de crianças na creche/total de funcionários na creche; crianças na mesma sala de aula: média de crianças que conviviam na creche na mesma sala de aula; crianças na mesma sala de descanso: média de crianças que conviviam na creche na mesma sala de descanso; ECPAE: Escore de capacidade de processamento da alimentação escolar.

O segundo, uma pesquisa de âmbito nacional, com coleta de informações em 147 instituições de educação infantil em seis capitais brasileiras, que revelou níveis de qualidade insatisfatórios 
nos espaços físicos das creches ${ }^{18}$. Os resultados desta pesquisa somam-se aos anteriores, destacando problemas de estrutura física e de recursos humanos, os quais são importantes para a implementação de práticas de cuidado adequadas no contexto das creches. Adicionalmente, nem todas as creches apresentaram condições propicias relacionadas à existência de merendeira e à disponibilidade de equipamentos na cozinha, com possíveis prejuízos na elaboração das refeições e na garantia da alimentação escolar.

Por outro lado, problemas importantes foram encontrados em relação ao desenvolvimento de atividades que devem integrar as ações no contexto do Programa Nacional de Alimentação Escolar. Nesse sentido, indicaram-se falhas relacionadas à existência de hortas escolares, ao desenvolvimento de ações de saúde, à participação dos pais e à inserção de atividades de alimentação e nutrição por parte dos professores nas suas práticas diárias. Embora sejam poucos os estudos na literatura com abordagens similares à do presente, observações realizadas em outras localidades do país têm apontado problemas semelhantes ${ }^{14,19,20}$. Essas condições podem estar relacionadas a uma concepção errada do Programa e à falta de profissionais capacitados na sua gestão e execução, como sugerido em outros estudos ${ }^{14,19}$.

Nesse sentido, cabe ressaltar a importância do envolvimento de todos os atores (nutricionistas, merendeiras, membros dos Conselhos de Alimentação Escolar, educadores, coordenadores pedagógicos) no sentido de aperfeiçoar o controle social e a execução do Programa Nacional de Alimentação Escolar ${ }^{21}$. Destaca-se, para esses fins, a relevância de ampliar e fortalecer os Conselhos de Alimentação Escolar, de incorporar efetivamente nutricionistas qualificados no desenvolvimento das ações, de treinar os profissionais da área de educação para sua adequação aos objetivos do Programa e de fortalecer as ações de educação alimentar e nutricional com o envolvimento de todos os membros da comunidade ${ }^{2,22-28}$. Esses aspectos são imprescindíveis para que o Programa Nacional de Alimentação Escolar deixe de ter uma visão reducionista, associada ao fornecimento de refeições, e passe a efetivamente incorporar uma concepção mais ampla ligada à efetivação do direito humano à alimentação adequada, da segurança alimentar e nutricional e da promoção da alimentação saudável, como processo de ressignificação implícito nas alterações do arcabouço legal do Programa ${ }^{20,22,27,29-31}$.

No contexto da educação alimentar e nutricional, deve-se destacar a importância do "Marco de Referência de Educação Alimentar e Nutricional para Políticas Públicas" 32 por suas especificidades como documento de orientação de conceitos, princípios e diretrizes para o desenvolvimento de práticas de educação referenciadas mais qualificadas ${ }^{33}$. Nesse sentido, ressalta-se a importância da avaliação das ações, considerando que o campo de atuação da educação alimentar e nutricional ainda não está claramente definido, e a compreensão do impacto como tema de pesquisa pouco abordado que deve ser priorizado 9,19,33,34.

Em relação ao perfil das merendeiras, os resultados deste estudo mostraram variedades de condições pessoais e de formas de relação de trabalho, as quais poderiam estar relacionadas às, igualmente, múltiplas condições encontradas no que se refere às funções, satisfação e interesses do ponto de vista profissional. Por outro lado, coincidindo com os resultados do presente trabalho, os estudos sinalizam pontos positivos como a valorização, a humanização e o interesse das merendeiras em relação ao seu trabalho, incluindo a vontade de capacitar-se e o prazer de cozinhar para as crianças ${ }^{14,25,35,36}$.

Esses aspectos, além das possíveis repercussões positivas na qualidade das refeições, colocam as merendeiras como atores importantes no processo de transformação da visão do Programa e na consolidação da educação em saúde como eixo do mesmo ${ }^{23,37,38}$. Nesse sentido, destaca-se a importância de um olhar mais atento para as potencialidades desse profissional, que considere as necessidades relacionadas ao seu processo de trabalho, como garantias estruturais, suporte técnico e vínculos com os outros profissionais ${ }^{25,35-37}$. Esses são fatores relevantes para que características individuais das merendeiras expressem-se com menor intensidade e que possa prevalecer o bom desempenho das funções em sintonia com a proposta e os objetivos do Programa Nacional de Alimentação Escolar.

No centro de atenção do presente trabalho, o estado nutricional das crianças, principalmente por meio do índice Estatura/Idade, mostrou diferenças estatísticas para várias das características das creches consideradas nas análises. Em geral, essas características refletem condições de estrutura relacionadas com a capacidade de recursos humanos para prestar atenção às crianças e com o modo de convivência em termos de aglomeração. Sugere-se, portanto, que o estado nutricional das crianças que frequentam creches pode ser influenciado por essas circunstâncias. Assim, adverte-se que as condições antepostas podem proteger a situação nutricional e de saúde, mani- 
festando-se a importância do cumprimento das normas que regulamentam aspectos nesse sentido $^{7-9}$. Teoricamente, a relevância desses fatores fica implícita no conceito de efetividade, uma vez que este vincula os efeitos aos recursos, atividades e produtos envolvidos ${ }^{39}$.

Alguns estudos têm abordado os diferenciais no estado nutricional ou na situação de saúde em relação às condições e à atenção prestada por diferentes serviços ${ }^{40-45}$, similarmente aos resultados desta pesquisa no contexto específico das creches. $\mathrm{O}$ fato de a capacidade de processamento da alimentação escolar não ter apresentado diferenças nas médias dos índices do estado nutricional, sugere, talvez, que indicadores simples, e não compostos, expressem melhor as relações em discussão.

A melhor condição nutricional de Estatura/ Idade das crianças com frequência à creche em tempo total quando comparadas com aquelas em tempo parcial, segundo os resultados observados neste trabalho, converge para os conhecimentos expostos na literatura ${ }^{2,10,46}$. Esse resultado pode ter explicações em dois ângulos. Por um lado, considerando o papel protetor das creches no desenvolvimento físico, psicológico, intelectual e social. Por outro, ponderando a oportunidade que refeições gratuitas e planejadas por profissionais preparados oferecem às crianças de famílias vulneráveis a em estados de insegurança alimentar ${ }^{2,47}$.

A zona rural, como ambiente de exposição em relação ao crescimento linear das crianças de Campina Grande, converge para os resultados de estudos populacionais ${ }^{48,49}$. Esses achados mostram a necessidade de se continuar cuidando dos problemas que permeiam as vidas nas zonas rurais, apesar da crescente urbanização do Brasil ${ }^{50}$. Além disso, colocam em discussão possíveis diferenças de estrutura, de procedimentos e de conscientização que podem estar condicionados pela localização geográfica, as quais devem ser consideradas na qualificação dos cuidados de saúde ${ }^{40}$.

Alguns fatores relacionados ao estado nutricional das crianças não foram considerados neste estudo, como as condições familiares ligadas à situação socioeconômica e de segurança alimentar. Nesse sentido, há que destacar as evidências da literatura que mostram, nas crianças menores de cinco anos, as melhorias nas condições socioeconômicas como um dos fatores mais importantes relacionados aos ganhos no estado nutricional ${ }^{51}$ e menores médias de Estatura/Idade e Peso/Estatura nos casos de insegurança alimentar ${ }^{52}$. Na conjuntura descrita, os resultados do presente estudo devem ser interpretados com cautela, pois as condições antepostas não foram analisadas, sendo questionado se a estrutura das creches não representaria um possível fator de confundimento, aspecto que não foi contemplado no desenho do presente estudo. Ainda, deve ser sustentada a hipótese de que crianças desnutridas têm acesso às creches com pior qualidade estrutural e de recursos humanos, o que pode influenciar negativamente o estado nutricional, já comprometido pela vulnerabilidade socioeconômica. Nessa situação, creches de baixa qualidade teriam maior dificuldade em atuar como promotoras de saúde e seriam as frequentadas por crianças mais vulneráveis.

Como possível limitação deste estudo podese destacar o desenho transversal, que não permite inferir relações de causa e efeito. Além disso, o uso de balança com graduação em 100g para as crianças menores de dois anos pode levar a interpretações equivocadas do estado antropométrico.

\section{Conclusão}

As creches apresentam níveis de qualidade insatisfatórios relacionados ao desenvolvimento de atividades que devem integrar o Programa Nacional de Alimentação Escolar. Comprometidos, constatam-se, também, alguns itens estruturais relacionados à capacidade dos recursos humanos para prestar atenção às crianças e aos modos de convivência (aglomeração), os quais podem marcar diferenças no estado nutricional destas. Contrariamente, a frequência da criança à creche em tempo integral e a urbanização predispõem positivamente o crescimento das crianças. O planejamento e a execução de outras pesquisas similares são urgentes para uma melhor compreensão do fenômeno em questão e a tomada de decisões oportunas relacionadas às medidas da política educacional. 


\section{Referências}

1. Oliveira JS, Lira PIC, Carvalho AGC, Barros MFA, Lima MC. Fatores associados ao estado nutricional em crianças de creches públicas do município de Recife, PE, Brasil. Rev Bras Epidemiol 2013; 16(2):502512.

2. Goulart RMM, Banduk MLS, Taddei JAAC. Uma revisão das ações de nutrição e do papel do nutricionista em creches. Rev Nutr 2010; 23(4):655-665.

3. Biscegli TS, Romera J, Candido AB, Santos JM, Candido ECA, Binotto AL. Estado nutricional e prevalência de enteroparasitoses em crianças matriculadas em creche. Rev Paul Pediatr 2009; 27(3):289-295.

4. Azevedo MMS, Cabral PC, Diniz AS, Fisberg M, Fisberg RM, Arruda IKG. Deficiência de vitamina A em pré-escolares da cidade do Recife, Nordeste do Brasil. Arch Latinoam Nut 2010; 60(1):36-41.

5. Bogus CM, Nogueira-Martins MCF, Moraes DEB, Taddei JAA. Cuidados oferecidos pelas creches: percepções de mães e educadoras. Rev Nutr 2007; 20(5):499414.

6. Barros RP, Carvalho M, Franco S, Mendonça S, Rosalém A. Uma avaliação do impacto da qualidade da creche no desenvolvimento infantil. PPE 2011; 41(2):213-232.

7. Vasconcelos RM, Tancredi RCP, Marin VA. Políticas e normativas aplicadas às creches municipais do Rio de Janeiro. Cien Saude Colet 2013; 18(11):3281-3290.

8. Brasil. Ministério da Solidariedade e da Segurança Social. Portaria n. ${ }^{\circ}$ 262/2011 de 31 de agosto. Diário da República 2011; 31 ago.

9. Brasil. Ministério da Educação. Fundo Nacional de Desenvolvimento da Educação. Resolução no 26, de 17 de junho de 2013. Dispõe sobre o atendimento da alimentação escolar aos alunos da educação básica no âmbito do Programa Nacional de Alimentação Escolar - PNAE. Diário Oficial da União 2013; 18 jun.

10. Pereira AS, Lanzillotti HS, Soares EA. Frequência à creche e estado nutricional de pré-escolares: uma revisão sistemática. Rev Paul Pediatr 2010; 28(4):366372.

11. Leroy JL, Gadsden P, Guijarro M. The impact of daycare programs on child health, nutrition and development in developing countries: a systematic review. Washington: International Food Policy Research Institute; 2011.

12. Figueroa Pedraza D, Queiroz D, Menezes TN. Segurança alimentar em famílias com crianças matriculadas em creches públicas do estado da Paraíba, Brasil. Rev Nutr 2013; 26(5):517-527.

13. Instituto Brasileiro de Geografia e Estatística (IBGE). Pesquisa Nacional por Amostra de Domicílios: Segurança Alimentar 2004. Rio de Janeiro: IBGE; 2006.

14. Figueroa Pedraza D, Andrade SLSS, Monteiro JS, Lira PIC. Avaliação do Programa de Alimentação Escolar municipal de Olinda - Pernambuco. RBPS 2007; 20(2):76-85.

15. World Health Organization (WHO). Physical status: the use and interpretation of anthropometry. Geneva: WHO; 1995. (WHO - Technical Report Series, 854).
16. World Health Organization (WHO). WHO Child Growth Standards. Length/height-for-age, weight-forage, weight-for-length, weight-for-height and body mass index-for-age. Methods and development. Geneva: WHO; 2006.

17. Campos MM, Füllgraf J, Wiggers V. A qualidade da educação infantil brasileira: alguns resultados de pesquisa. Cadernos Pesquisa 2006; 36(127):87-128.

18. Campos MM, Esposito YL, Bhering E, Gimenes N, Abuchaim B. A qualidade da educação infantil: um estudo em seis capitais brasileiras. Cadernos Pesquisa 2011; 41(142):20-54.

19. Silva CAM, Marques LA, Bonomo E, Bezerra OMPA, Corrêa MS, Passos LSF, Souza AA, Barros BF, Souza DMS, Reis JA, Andrade NG. O Programa Nacional de Alimentação Escolar sob a ótica dos alunos da rede estadual de ensino de Minas Gerais, Brasil. Cien Saude Colet 2013; 18(4):963-969.

20. Machado PMO, Machado MS, Schmitz BAS, Corso ACT, González-Chica DA, Vasconcelos FAG. Caracterização do Programa Nacional de Alimentação Escolar no Estado de Santa Catarina. Rev Nutr 2013; 26(6):715-725.

21. Santos LAS, Paiva JB, Mello AL, Fontes GAV, Sampaio LR, Freitas MCS. O nutricionista no programa de alimentação escolar: avaliação de uma experiência de formação a partir de grupos focais. Rev Nutr 2012; 25(1):107-117.

22. Peixinho AML. A trajetória do Programa Nacional de Alimentação Escolar no período de 2003-2010: relato do gestor nacional. Cien Saude Colet 2013; 18(4):909916.

23. Mello AL, Vidal Júnior PO, Sampaio LR, Santos LAS, Freitas MCS, Fontes GAV. Perfil do nutricionista do programa nacional de alimentação escolar na região Nordeste do Brasil. Rev Nutr 2012; 25(1):119-132.

24. Chaves LG, Santana TCM, Gabriel CG, Vasconcelos FA. Reflexões sobre a atuação do nutricionista no Programa Nacional de Alimentação Escolar no Brasil. Cien Saude Colet 2013; 18(4):917-926.

25. Fernandes AGS, Fonseca ABC, Silva AA. Alimentação escolar como espaço para educação em saúde: percepção das merendeiras do município do Rio de Janeiro, Brasil. Cien Saude Colet 2014; 19(1):39-48.

26. Gabriel CG, Machado MS, Schmitz BAS, Corso ACT, Caldeiras GV, Vasconcelos FAG. Conselhos Municipais de Alimentação Escolar em Santa Catarina: caracterização e perfil de atuação. Cien Saude Colet 2013; 18(4):971-978.

27. Bandeira LM, Chagas CMS, GUBERT MB, Toral N, Monteiro RA. Análise dos pareceres conclusivos dos Conselhos de Alimentação Escolar sobre a execução do Programa Nacional de Alimentação Escolar. Rev Nutr 2013; 26(3):343-351.

28. Juzwiak CR, Castro PM, Batista SHSS. A experiência da Oficina Permanente de Educação Alimentar e em Saúde (OPEAS): formação de profissionais para a promoção da alimentação saudável nas escolas. Cien Saude Colet 2013; 18(4):1009-1018. 
29. Teo CRPA, Monteiro CA. Marco legal do Programa Nacional de Alimentação Escolar: uma releitura para alinhar propósitos e práticas na aquisição de alimentos. Rev Nutr 2012; 25(5):657-668.

30. Vasconcelos FAG. Programa nacional de alimentação escolar: limites e possibilidades para a garantia do direito humano à alimentação adequada, saudável e sustentável. Cien Saude Colet 2013; 18(4):906.

31. Siqueira RL, Cotta RMM, Ribeiro RCL, Sperandio N, Priore SE. Análise da incorporação da perspectiva do Direito Humano à Alimentação Adequada no desenho institucional do Programa Nacional de Alimentação Escolar. Cien Saude Colet 2014; 19(1):301310.

32. Brasil. Ministério do Desenvolvimento Social e Combate à Fome (MDS). Marco de referência de educação alimentar e nutricional para as políticas públicas. Brasília: MDS; 2012.

33. Amparo-Santos L. Avanços e desdobramentos do marco de referência da educação alimentar e nutricional para políticas públicas no âmbito da universidade e para os aspectos culturais da alimentação. Rev Nutr 2013; 26(5):595-600.

34. Ramos FP, Santos LAS, Reis ABC. Educação alimentar e nutricional em escolares: uma revisão de literatura. Cad Saude Publica 2013; 29(11):2147-2161.

35. Miron VR, Stefanello CL, Mattos KM, Colome JS, Costenaro R, Carpes AD. Profissão merendeira: perfil profissional e condições socioeconômicas. Disciplinarum Scientia 2009; 10(1):87-95.

36. Carvalho AT, Muniz VM, Gomes JF, Samico I. Programa de alimentação escolar no município de João Pessoa - PB, Brasil: as merendeiras em foco. Interface (Botucatu) 2008; 12(27):823-834

37. Teo CRPA, Sabedot FRB, Schafer E. Merendeiras como agentes de educação em saúde da comunidade escolar: potencialidades e limites. Rev Esp Saude 2010; 11(2):11-20.

38. Leite CL, Cardoso RCV, Góes JAW, Figueiredo KVNA, Silva EO, Bezerril MM, Vidal Júnior PO, Santana AAC. Formação para merendeiras: uma proposta metodológica aplicada em escolas estaduais atendidas pelo programa nacional de alimentação escolar, em Salvador, Bahia. Rev Nutr 2011; 24(2):275-285.

39. Scarparo ALS, Oliveira VR, Bittencourt JMV, Ruiz ENF, Fernandes PF, Zys JZ, Moulin CC. Formação para nutricionistas que atuam no Programa Nacional de Alimentação Escolar: uma avaliação da efetividade. Cien Saude Colet 2013; 18(4):1001-1008.

40. Rocha ACD, Pedraza DF. Acompanhamento do crescimento infantil em unidades básicas de saúde da famí lia do município de Queimadas, Paraíba, Brasil. Texto Cont Enferm 2013; 22(4):1169-1178.

41. Azevedo MMS, Cabral PC, Diniz AS, Fisberg M, Fisberg RM, Arruda IKG. Deficiência de vitamina A em pré-escolares da cidade do Recife, Nordeste do Brasil. Arch Latinoam Nut 2010; 60(1):36-41.
42. Bernardi JR, Cesaro C, Fisberg RM, Fisberg M, Rodrigues GP, Vitolo MR. Consumo alimentar de micronutrientes entre pré-escolares no domicílio e em escolas de educação infantil do município de Caxias do Sul (RS). Rev Nutr 2011; 24(2):253-261.

43. Barbosa RM, Soares EA, Lanzillotti HS. Avaliação da ingestão de nutrientes de crianças de uma creche filantrópica: aplicação do consumo dietético de referência. Rev Bras Saude Matern Infant 2007; 7(2):159 166.

44. Carvalho MF, Lira PIC, Romani SAM, Santos IS, Veras AACA, Batista FM. Acompanhamento do crescimento em crianças menores de um ano: situação nos serviços de saúde em Pernambuco, Brasil. Cad Saude Publica 2008; 24(3):675-685.

45. Figueroa Pedraza D, Queiroz D, Sales MC. Doenças infecciosas em crianças pré-escolares brasileiras assistidas em creches. Cien Saude Colet 2014; 19(2):511528.

46. Figueroa Pedraza D, Souza MM, Rocha ACD. Fatores associados ao estado nutricional de crianças pré-escolares brasileiras assistidas em creches públicas: uma revisão sistemática. Rev Nutr 2015; 28(4):451-463.

47. Peixinho A, Balaban D, Rimkus L, Schwartzman F, Galante AP. Alimentação Escolar no Brasil e nos Estados Unidos. O Mundo da Saúde 2011; 35(2):128-136.

48. Brasil. Ministério da Saúde (MS). Pesquisa nacional sobre demografia e saúde da criança e da mulher. Brasília: MS; 2008.

49. Leal VL, Lira PIC, Menezes RCI, Oliveira JS, Sequeira SLA, Andrade SLS, Batista Filho M. Fatores associados ao declínio do déficit estatural em crianças e adolescentes em Pernambuco. Rev Saude Publica 2012; 46(2):234-241.

50. Victora CG, Aquino EML, Leal MC, Monteiro CA Barros FC, Szwarcwald CL. Saúde no Brasil 2. Saúde de mães e crianças no Brasil: progressos e desafios. [acessado 2015 abr 5]. Disponível em: http://portal. saude.gov.br/portal/arquivos/pdf/revista_the_lancet. pdf

51. Barata RB. Epidemiologia e políticas públicas. Rev Bras Epidemiol 2013; 16(1):3-17.

52. Santos LPD, Gigante DP. Relação entre insegurança alimentar e estado nutricional de crianças brasileiras menores de cinco anos. Rev Bras Epidemiol 2013; 16(4):984-994.

Artigo apresentado em 27/03/2015

Aprovado em 23/10/2015

Versão final apresentada em 25/10/2015 
